\title{
Symptomatic IgG3 deficiency successfully treated with intravenous immunoglobulin therapy
}

\author{
J.A. Snowden, A. Milford-Ward and J.T. Reilly \\ Northern General Hospital, Herries Road, Sheffield S5 7AU, UK
}

\begin{abstract}
Summary: We report the case of a 35 year old female who presented with an 8 year history of repeated upper respiratory tract infection, lymphadenopathy and sinusitis associated with IgG3 deficiency. Courses of monthly intravenous immunoglobulin therapy $(0.4 \mathrm{~g} / \mathrm{kg})$ resulted in a dramatic clinical improvement on three different occasions. We suggest that investigation of adults with features of immunosuppression, despite normal levels of total IgG, should include IgG3 subclass analysis and that symptomatic patients should be given a trial of immunoglobulin replacement therapy.
\end{abstract}

\section{Introduction}

The occurrence of selective deficiencies of the four IgG subclasses ${ }^{1}$ has been recognized since the 1960 s but routine testing has only become available to hospital laboratories relatively recently. IgG subclass deficiency may be present even if total IgG levels are normal and, unless specifically requested, IgG subclass deficiency may be overlooked. We present a case history to illustrate the value of considering IgG subclass deficiency in the diagnosis of recurrent infection and discuss how such patients may be investigated and treated.

\section{Case report}

A 35 year old woman was referred to our unit with a long history of recurrent upper respiratory tract infections (coryza, sore throat, sinusitis), with associated lymphadenopathy, sweats, fever and headaches. On average, each episode occurred for several days a month, often causing time off work. In addition, she had a history of recurrent vaginal candidiasis, urinary tract infections and a year prior to referral had suffered an episode of pelvic inflammatory disease. Over the previous 8 years these symptoms had been extensively investigated by her general practitioners and by three hospital physicians. No underlying cause had been identified and her symptoms were labelled by one physician as 'psychosomatic'. At one stage, she was treated for depression.

Subsequent investigations revealed a greatly reduced IgG subclass 3 (IgG3) level of $<0.06 \mathrm{~g} / 1$

Correspondence: J.T. Reilly, M.R.C.P., M.R.C.Path. Accepted: 25 May 1994 (normal range $0.2-1.9 \mathrm{~g} / \mathrm{l}$ ) with other $\mathrm{IgG}$ subclasses in the normal range and normal IgG levels on five out of six occasions, the exception being a borderline level of $5.2 \mathrm{~g} / 1$ (normal range $5.4-16 \mathrm{~g} / 3$ 1). IgM and IgA levels were normal, along with full blood count, renal and liver function, blogi $\overrightarrow{0}$ glucose, autoantibodies, complement, chest X-ra and paranasal sinus X-ray. Lymphocyte subst. analysis showed an increase in activated $\mathrm{T}$ lym-응 phocytes, consistent with viral infection, but a normal CD4 count. However, broad serological studies including Epstein-Barr virus, cytomegalovirus, toxoplasma and upper respiratory $\stackrel{\Phi}{\varnothing}$ viruses were negative. Functional immunoglobulin $\overrightarrow{\vec{O}}$ response was assessed prior to and 2 weeks post- 3 immunization with pneumococcal vaccine. IgG subclass levels and specific anti-pneumococcal antibody titres were not changed by vaccination $\overline{0}$ (total anti-pneumococcal IgG pre-vaccination $\underline{3}$. $<1: 8$, post-vaccination $<1: 8$, reference range for $\frac{\sigma}{3}$ increment in adults $=75-5,250$, mean $=579^{2}$ ),

A trial of intravenous immunoglobulin therapy $\delta$ ('Gaminume-N' (Cutter)) given monthly at a dose of $0.4 \mathrm{~g} / \mathrm{kg}$ was followed by a striking absence of $\frac{\mathrm{O}}{2}$ further episodes of upper respiratory, sinus or $D$ urinary tract infections in the following 6 months. After this period a trial without immunoglobulin $\tilde{N}^{\circ}$ replacement was attempted but within 6 weeks the patient reported a recurrence of the original upper $\tilde{D}$ respiratory tract symptoms. Treatment was recom- $\underset{\omega}{ }$ menced to good effect. We have tried on two further occasions to withdraw treatment and each 0 time the symptoms returned within 2 months. Immunoglobulin subclasses have been assayed $\stackrel{?}{+}$ prior to and during regular replacement with a $\frac{0}{2}$ four-fold increase in IgG3 to well within the normal $\underset{\mathbb{D}}{\vec{D}}$

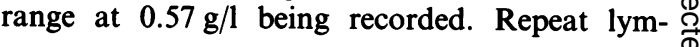


phocyte subsets taken whilst the patient was well and on regular replacement therapy showed disappearance of the activated lymphocytes (suggestive of successful eradication of the probable virus).

Other family members were assessed. Her parents and brother have no history of recurrent infection and have IgG subclasses in the normal range. However, her 4 year old daughter has a reduced level of IgG 3 at $0.16 \mathrm{~g} / 1$ (age-specific range $0.3-1.0 \mathrm{~g} / \mathrm{l})$. The child suffers with recurrent tonsillitis four times per year but also has congenital heart disease (potentially an additional cause of immune compromise).

\section{Discussion}

IgG subclasses differ from each other structurally, by the nature of their 'constant' regions, and functionally. Antibody responses to protein antigens such as viral envelopes tend to involve IgG1 and IgG3, whereas responses to capsular polysaccharides tend to involve IgG2 or IgG1, and responses to repetitive stimulation predominantly IgG4, IgG3 and IgG1, in contrast to IgG2 and IgG4, fix complement, bind to the surface of platelets and monocytes, and can be found in the cytoplasm, as opposed to the surface, of B lymphocytes. IgG subclass deficiency has been extensively reviewed in the specialist literature. ${ }^{1}$ It occurs in isolation accounting for one quarter of patients with otherwise unexplained recurrent infections and in the context of primary and acquired syndromes of immunodeficiency. Selective deficiencies of IgG2, associated with infections with capsulated bacteria, and IgG3 are the commonest. Infections most often affect the upper and lower respiratory tracts, although we have recently reported recurrent lymphocytic meningitis associated with selective IgG3 deficiency. ${ }^{3}$

Assessment of patients in whom an isolated deficiency of an immunoglobulin subclass is discovered should include exclusion of other causes of recurrent infection, and consideration of the type of infection and its frequency. In our patient, the recurrent upper respiratory tract symptoms and lymphadenopathy in association with activated lymphocytes suggests a viral aetiology and accords with previous descriptions of IgG3-deficient patients. The recurrent urinary tract infections and vaginal candidiasis are not especially unusual in women because of anatomical factors, but also appear to have responded to replacement therapy. Serological tests (including the 'Monospot' test) may be negative if patients are unable to mount a sufficient antibody response and this possibly explains why we have been unable to identify a viral agent in our patient.

A functional immunoglobulin response ${ }^{1,2}$ is an essential investigation in any patient being assessed for immunoglobulin replacement and is analogous to dynamic testing in endocrinology. By definition, $5 \%$ of the normal healthy population have IgG subclass levels below the 'normal' range (defined as 5th to the 95th percentile) but, despite the quantitative deficiency, they are functionally competent and respond appropriately when challenged with antigen. In addition, there are occasional individuals with normal levels of IgG subclasses who are functionally immunodeficient in that they are unable to produce an increment in a specific IgG subclass or subclasses. Hence, immunization or challenge with an appropriate antigen is an important exercise in the investigation of a putative IgG subclass deficiency, and in the wider context of the full investigation of a patient with a history suggestive of immune deficiency but with ostensibly normal immunoglobulin levels. Pneumococcal vaccine ('Pneumovax II' (Morson)) is an inexpensive and readily available preparation that 'stresses' all four IgG subclasses, ${ }^{1,2}$ although other agents, including tetanus toxoid, haemophilus and influenza vaccines and rhesus $D$ antigen, may also be used. In our patient the low IgG3 concentration on initial screening was a marker for an abnormal functional immunoglobulin response not only for the IgG3 subclass but also for IgG overall. To complete the investigations, family studies should be performed to establish the familial nature of the deficiency, and to offer assessment and treatment to other family members who may suffer recurrent infection.

Once fully assessed the patient should be given the option of a therapeutic trial of intravenous immunoglobulin. The efficacy of immunoglobulin replacement has long been recognized in patients with low immunoglobulin levels and a randomized, controlled trial showed reduction in the incidence of recurrent bronchitis in patients with IgG3 levels less than $0.41 \mathrm{~g} / \mathrm{l}^{4}$ This is usually given as an intravenous infusion at $0.4 \mathrm{~g} / \mathrm{kg}$ once per month and requires half day hospital attendance, although highly motivated patients can be considered for home treatment. Various preparations exist (e.g. 'Gamimune-N' (Cutter), 'Sandoglobulin' (Sandoz), 'Venoglobulin' (Alpha), 'Endobulin' (Immuno), 'Vigam' (BPL)) and these differ in their proportions of the IgG subclasses. The choice should depend on which IgG subclass or subclasses are deficient. For example, our patient received 'Gamimune N', which is relatively rich in IgG3. Early reports associated pooled immunoglobulin with non-A non-B hepatitis but these risks are considered no longer to exist with modern, virally inactivated preparations. ${ }^{5}$ However, before commencing treatment the patient should be adequately counselled that they are receiving a blood product. 
A decision should therefore be arrived at jointly by the clinician and the patient weighing up the potential benefits of relief from infection against the inconvenience of attending hospital on a monthly basis. In contrast to the case presented, the patient with three episodes of lymphocytic meningitis over 5 years declined regular prophylaxis. ${ }^{3}$ The trial should last for at least 6 months and the patient should keep a log of intercurrent infections. If successful, the treatment should be continued indefinitely. However, before committing the

\section{References}

1. Jefferis, R. \& Kumararatne, D.S. Selective IgG subclass deficiency: quantitation and clinical relevance. Clin Exp Immunol 1990, 81: 357-367.

2. Hazelwood, M., Nusrat, R., Kumararatne, D.S. et al. The acquisition of anti-pneumococcal capsular polysaccharide. Haemophilus influenzae type b and tetanus toxoid antibodies, with age, in the UK. Clin Exp Immunol 1993, 93: 157-164.

3. Snowden, J.A., Milford-Ward, A., Cookson, L.J. \& McKendrick, M.W. Recurrent lymphocytic meningitis associated with isolated IgG subclass 3 deficiency. J Infect 1993, 27: 285-289. patient to a lifetime of therapy, the clinician may want to have one or more trials off treatment to confirm the benefits. It is desirable to check that a sufficient increment in the deficient subclass is achieved and, if necessary, increase the dose or frequency of the immunoglobulin preparation appropriately. The cost of the treatment depends on the preparation and the patient's weight but, regrettably, is never cheap. In the case presented the cost of one year's treatment is in the region of $£ 5,000$.

4. Soderstrom, T., Soderstrom, R. \& Enskog, A. Immunoglobulin subclasses and prophylactic use of immunoglobulin in immunoglobulin G subclass deficiency. Cancer 1991, 68: $1426-1429$.

5. Suomela, H. Inactivation of viruses in blood and plasma products. Transfus Med Rev 1993, 7: 42-57. 